\title{
Energy Solution to the Chern-Simons-Schrödinger Equations
}

\author{
Hyungjin Huh \\ Department of Mathematics, Chung-Ang University, Seoul 156-756, Republic of Korea
}

Correspondence should be addressed to Hyungjin Huh; huh@cau.ac.kr

Received 19 November 2012; Accepted 17 January 2013

Academic Editor: Graziano Crasta

Copyright (C) 2013 Hyungjin Huh. This is an open access article distributed under the Creative Commons Attribution License, which permits unrestricted use, distribution, and reproduction in any medium, provided the original work is properly cited.

We prove that the Chern-Simons-Schrödinger system, under the condition of a Coulomb gauge, has a unique local-in-time solution in the energy space $H^{1}\left(\mathbb{R}^{2}\right)$. The Coulomb gauge provides elliptic features for gauge fields $A_{0}$, $A_{j}$. The Koch- and Tzvetkov-type Strichartz estimate is applied with Hardy-Littlewood-Sobolev and Wente's inequalities.

\section{Introduction}

We study herein the initial value problem of the ChernSimons-Schrödinger (CSS) equations

$$
\begin{gathered}
i D_{0} \phi+D_{j} D_{j} \phi=-\lambda|\phi|^{2} \phi, \\
\partial_{0} A_{1}-\partial_{1} A_{0}=-\operatorname{Im}\left(\bar{\phi} D_{2} \phi\right), \\
\partial_{0} A_{2}-\partial_{2} A_{0}=\operatorname{Im}\left(\bar{\phi} D_{1} \phi\right), \\
\partial_{1} A_{2}-\partial_{2} A_{1}=-\frac{1}{2}|\phi|^{2},
\end{gathered}
$$

where $i$ denotes the imaginary unit; $\partial_{0}=\partial / \partial t, \partial_{1}=\partial / \partial x_{1}$, and $\partial_{2}=\partial / \partial x_{2}$ for $\left(t, x_{1}, x_{2}\right) \in \mathbb{R}^{1+2} ; \phi: \mathbb{R}^{1+2} \rightarrow \mathbb{C}$ is the complex scalar field; $A_{\mu}: \mathbb{R}^{1+2} \rightarrow \mathbb{R}$ is the gauge field; $D_{\mu}=\partial_{\mu}+i A_{\mu}$ is the covariant derivative for $\mu=0,1,2$, and $\lambda>0$ is a coupling constant representing the strength of interaction potential. The summation convention used involves summing over repeated indices and Latin indices are used to denote 1,2 .

The CSS system of equations was proposed in $[1,2]$ to deal with the electromagnetic phenomena in planar domains, such as the fractional quantum Hall effect or hightemperature superconductivity. We refer the reader to [3, 4] for more information on the physical nature of these phenomena.
The CSS system exhibits conservation of mass

$$
M(t)=\int_{\mathbb{R}^{2}}|\phi(t, x)|^{2} d x=M(0),
$$

and the conservation of total energy

$$
E(t)=\int_{\mathbb{R}^{2}}\left|D_{j} \phi(t, x)\right|^{2}-\frac{\lambda}{2}|\phi(t, x)|^{4} d x=E(0) .
$$

Note that the terms $|F|^{2}=(1 / 2) F_{\mu \nu} F^{\mu \nu}$ are missing in (3) when compared to the Maxwell-Schrödinger equations studied in [5].

To figure out the optimal regularity for the CSS system, we observe that the CSS system is invariant under scaling:

$$
\begin{gathered}
\phi^{a}(t, x)=a \phi\left(a^{2} t, a x\right), \quad A_{j}^{a}(t, x)=a A_{j}\left(a^{2} t, a x\right), \\
A_{0}^{a}(t, x)=a^{2} A_{0}\left(a^{2} t, a x\right) .
\end{gathered}
$$

Therefore, the scaled critical Sobolev exponent is $s_{c}=0$ for $\phi$. In view of (2) we may say that the initial value problem of the CSS system is mass critical.

The CSS system is invariant under the following gauge transformations:

$$
\phi \longrightarrow \phi e^{i \chi}, \quad A_{\mu} \longrightarrow A_{\mu}-\partial_{\mu} \chi
$$

where $\chi: \mathbb{R}^{2+1} \rightarrow \mathbb{R}$ is a smooth function. Therefore, a solution to the CSS system is formed by a class of gauge 
equivalent pairs $\left(\phi, A_{\mu}\right)$. In this work, we fix the gauge by imposing the Coulomb gauge condition of $\partial_{j} A_{j}=0$, under which the Cauchy problem of the CSS system may be reformulated as follows:

$$
\begin{gathered}
i \partial_{t} \phi-A_{0} \phi+\Delta \phi+2 i A_{j} \partial_{j} \phi-A_{j}^{2} \phi=-\lambda|\phi|^{2} \phi, \\
\partial_{1} A_{2}-\partial_{2} A_{1}=-1 / 2|\phi|^{2}, \quad \partial_{1} A_{1}+\partial_{2} A_{2}=0, \\
\Delta A_{0}=\operatorname{lm}\left(Q_{12}(\bar{\phi}, \phi)\right)+\partial_{1}\left(A_{2}|\phi|^{2}\right)-\partial_{2}\left(A_{1}|\phi|^{2}\right),
\end{gathered}
$$

where the initial data $\phi(0, x)=\phi_{0}(x)$. For the formulation of (6)-(8) we refer the reader to Section 3.

The initial value problem of the CSS system was investigated in $[6,7]$. It was shown in [6] that the Cauchy problem is locally well posed in $H^{2}\left(\mathbb{R}^{2}\right)$, and that there exists at least one global solution, $\phi \in L^{\infty}\left(\mathbb{R}^{+} ; H^{1}\left(\mathbb{R}^{2}\right)\right) \cap C_{\omega}\left(\mathbb{R}^{+} ; H^{1}\left(\mathbb{R}^{2}\right)\right)$, provided that the initial data are made sufficiently small in $L^{2}\left(\mathbb{R}^{2}\right)$ by finding regularized equations. They also showed, by deriving a virial identity, that solutions blow up in finite time under certain conditions. Explicit blow-up solutions were constructed in [8] through the use of a pseudoconformal transformation. The existence of a standing wave solution to the CSS system has also been proved in $[9,10]$.

The adiabatic approximation of the Chern-SimonsSchrödinger system with a topological boundary condition was studied in [11], which provides a rigorous description of slow vortex dynamics in the near self-dual limit.

Taking the conservation of energy (3) into account, it seems natural to consider the Cauchy problem of the CSS system with initial data $\phi_{0} \in H^{1}\left(\mathbb{R}^{2}\right)$. Our purpose here is to supplement the original result of [6] by showing that there is a unique local- in-time solution in the energy space $H^{1}\left(\mathbb{R}^{2}\right)$. We follow a rather direct means of constructing the $H^{1}$ solution and prove the uniqueness. We adapt the idea discussed in $[12,13]$ where a low regularity solution of the modified Schrödinger map (MSM) was studied. In fact, the CSS and MSM systems have several similarities except for the defining equation for $A_{0}$. In the MSM, $A_{0}$ can be written roughly as $R_{j} R_{k}\left(u^{2}\right)+|u|^{2}$, where $R_{j}=\partial_{j}(-\Delta)^{-1 / 2}$ denotes the Riesz transform. The local existence of a solution to the MSM was proved in [12] for the initial data in $H^{s_{1}}\left(\mathbb{R}^{2}\right)$ with $s_{1}>1 / 2$, and similarly, the uniqueness was proved in [14] for $H^{s_{2}}\left(\mathbb{R}^{2}\right)$ with $s_{2}>3 / 4$. To show the existence and uniqueness of the $H^{1}$ solution to the CSS system, the estimate of the gauge field, $A_{0}$, is important for situations in which special structures of nonlinear terms in the defining equation for $A_{0}$ are used. The following describes are our main results.

Theorem 1. Let initial data $\phi_{0}$ belong to $H^{1}\left(\mathbb{R}^{2}\right)$. Then, there exists a local-in-time solution, $\phi$, to (6)-(8) that satisfies

$$
\begin{gathered}
\phi \in L^{\infty}\left([0, T) ; H^{1}\left(\mathbb{R}^{2}\right)\right) \cap C\left([0, T) ; L^{2}\left(\mathbb{R}^{2}\right)\right), \\
J^{\delta} \phi \in L^{p}\left(0, T ; L^{q}\left(\mathbb{R}^{2}\right)\right),
\end{gathered}
$$

where $0<\delta<1 / 2,2<\delta q, 1 / p+1 / q=1 / 2$ and $J=(1-\Delta)^{1 / 2}$.
Theorem 2. Let $\phi$ and $\psi$ be solutions to $(6)-(8)$ on $(0, T) \times$ $\mathbb{R}^{2}$ in the distribution sense with the same initial data to that outlined vide supra. Moreover, one assumes that

$$
\begin{gathered}
\phi, \psi \in C\left([0, T] ; L^{2}\left(\mathbb{R}^{2}\right)\right), \\
\|\phi\|_{L_{T}^{\infty} H^{1}} \leq M, \quad\|\psi\|_{L_{T}^{\infty} H^{1}} \leq M,
\end{gathered}
$$

for some constant $M>0$. One then has $\|(\phi-\psi)(t, \cdot)\|_{L^{2}\left(\mathbb{R}^{2}\right)}=$ 0 for $0 \leq t \leq T$.

We present some preliminaries in Section 2. Theorems 1 and 2 are proved in Sections 3 and 4, respectively. We conclude the current section by providing a few notations. We denote space time derivatives by $\partial=\left(\partial_{0}, \partial_{1}, \partial_{2}\right)$ and $\nabla$ is used for spacial derivatives. We use the standard Sobolev spaces $W^{s, p}$, with the norm $\|f\|_{W^{s, p}}=\left\|J^{s} f\right\|_{L^{p}}$ and $\dot{W}^{s, p}$ with the norm $\|f\|_{\dot{W}^{s, p}}=\left\||\nabla|^{s} f\right\|_{L^{p}}$, where $J=(1-\Delta)^{1 / 2}$ and $|\nabla|=(-\Delta)^{1 / 2}$. The space $H^{s}$ denotes $W^{s, 2}$. We define the space time norm as $\|f\|_{L_{T}^{p} L^{q}}=\left(\int_{0}^{T}\|f(t, \cdot)\|_{L^{q}\left(\mathbb{R}^{2}\right)}^{p} d t\right)^{1 / p}$. We use $c, C$ to denote various constants. Because we are interested in local solutions, we may assume that $T \leq 1$. Thus, we replace the smooth function of $T, C(T)$ with $C$. We also use the convention of writing $A \lesssim B$ as shorthand for $A \leq C B$.

\section{Preliminaries}

We collect here a few lemmas used for the proof of Theorems 1 and 2. The following lemma is reminiscent of Wente's inequality (see $[15,16])$.

Lemma 3. Let $f$ and $g$ be two functions in $H^{1}\left(\mathbb{R}^{2}\right)$ and let $u$ be the solution of

$$
\Delta u=\partial_{1} f \partial_{2} g-\partial_{2} f \partial_{1} g \text { in } \mathbb{R}^{2}
$$

where $u$ is small at infinity. Then, $u \in C\left(\mathbb{R}^{2}\right) \cap \dot{H}^{1}\left(\mathbb{R}^{2}\right)$ and

$$
\|u\|_{L^{\infty}\left(\mathbb{R}^{2}\right)}+\|\nabla u\|_{L^{2}\left(\mathbb{R}^{2}\right)} \leq C\|\nabla f\|_{L^{2}\left(\mathbb{R}^{2}\right)}\|\nabla g\|_{L^{2}\left(\mathbb{R}^{2}\right)} .
$$

The following energy estimate in $[17,18]$ is used for estimating a solution to the magnetic Schrödinger equation.

Lemma 4. Let $u$ be a solution of

$$
i \partial_{t} u+\Delta u+2 i \operatorname{div}(a u)=F,
$$

where $a=\left(a_{1}(t, x), a_{2}(t, x)\right)$ and $a_{j}$ are real-valued functions. Then, for $s \geq 0$ there exists an absolute constant $C_{s}>0$ such that

$$
\begin{aligned}
\|u(t, \cdot)\|_{\dot{H}^{s}} \leq & \|u(0, \cdot)\|_{\dot{H}^{s}} \\
& +C_{s} \int_{0}^{t}\left(\|\nabla a\|_{\dot{H}^{s}}\|u\|_{L^{\infty}}+\|\nabla a\|_{L^{\infty}}\|u\|_{\dot{H}^{s}}+\|F\|_{\dot{H}^{s}}\right) d s,
\end{aligned}
$$

wherein one means the homogeneous Sobolev space $\dot{H}^{s}$ when $s>0$ and simply $L^{2}$ when $s=0$. 
The following type of Strichartz estimate was used in [19, 20] for the study of the Benjamin-Ono equation. We refer to [12] for the counterpart to the Schrödinger equation.

Lemma 5. Let $T \leq 1$ and $v$ be a solution to the equation

$$
i \partial_{t} v+\Delta v=F_{1}+F_{2}, \quad(t, x) \in(0, T) \times \mathbb{R}^{2} .
$$

Then, for $\delta \in R$ and $\varepsilon>0$, one has

$$
\left\|J^{\delta} v\right\|_{L_{T}^{p} L^{q}} \leqslant\|v\|_{L_{T}^{\infty} H^{\delta+1 / 2+\varepsilon}}+\left\|F_{1}\right\|_{L_{T}^{2} H^{\delta-1 / 2}}+\left\|F_{2}\right\|_{L_{T}^{1} H^{\delta}}
$$

where $1 / p+1 / q=1 / 2$ and $2 \leq q<\infty$.

We use the following Gagliardo-Nirenberg inequality with the specific constant [21], especially for the proof of Theorem 2.

Lemma 6. For $2 \leq q<\infty$, one has

$$
\|u\|_{L^{q}\left(\mathbb{R}^{2}\right)} \leq(4 \pi)^{(2-q) / 2 q}\left(\frac{q}{2}\right)^{1 / 2}\|u\|_{L^{2}\left(\mathbb{R}^{2}\right)}^{2 / q}\|\nabla u\|_{L^{2}\left(\mathbb{R}^{2}\right)}^{1-2 / q} .
$$

\section{The Proof of Theorem 1}

Theorem 1 is proved in this section. Because the local wellposedness for smooth data is already known in [6], we simply present an a priori estimate for the solution to (6)-(8). Let us first explain (8). To derive it, note the following identities:

$$
\begin{aligned}
\overline{D_{\alpha} \phi} D_{\beta} \phi-\overline{D_{\beta} \phi} D_{\alpha} \phi= & Q_{\alpha \beta}(\bar{\phi}, \phi) \\
& -i\left(A_{\alpha} \partial_{\beta}\left(|\phi|^{2}\right)-A_{\beta} \partial_{\alpha}\left(|\phi|^{2}\right)\right), \\
D_{\alpha} D_{\beta} \phi & -D_{\beta} D_{\alpha} \phi=i F_{\alpha \beta} \phi
\end{aligned}
$$

where $Q_{\alpha \beta}(\bar{\phi}, \phi)=\partial_{\alpha} \bar{\phi} \partial_{\beta} \phi-\partial_{\beta} \bar{\phi} \partial_{\alpha} \phi$ and $F_{\alpha \beta}=\partial_{\alpha} A_{\beta}-\partial_{\beta} A_{\alpha}$. Note that the second-order terms $\partial_{\alpha \beta} \phi$ are cancelled out. Combined with the above algebra, the equation for $A_{0}$ comes from the second and third equations in (1):

$$
\begin{aligned}
\Delta A_{0} & =\partial_{1} \operatorname{Im}\left(\bar{\phi} D_{2} \phi\right)-\partial_{2} \operatorname{Im}\left(\bar{\phi} D_{1} \phi\right) \\
& =\operatorname{Im}\left(Q_{12}(\bar{\phi}, \phi)\right)+\partial_{1}\left(A_{2}|\phi|^{2}\right)-\partial_{2}\left(A_{1}|\phi|^{2}\right) .
\end{aligned}
$$

We then have the formulation (6)-(8) in which $\phi$ is the only dynamical variable and $A_{1}, A_{2}$, and $A_{0}$ are determined through (7) and (8).

The constraint equation $\partial_{1} A_{2}-\partial_{2} A_{1}=-1 / 2|\phi|^{2}$ and the Coulomb gauge condition $\partial_{1} A_{1}+\partial_{2} A_{2}=0$ provide an elliptic feature of $A=\left(A_{1}, A_{2}\right)$; that is, the components $A_{j}$ can be determined from $\phi$ by solving the elliptic equations

$$
\Delta A_{1}=\partial_{2}\left(\frac{1}{2}|\phi|^{2}\right), \quad \Delta A_{2}=-\partial_{1}\left(\frac{1}{2}|\phi|^{2}\right) .
$$

Taking into account that the Coulomb gauge condition in Maxwell dynamics deduces a wave equation, the previous observation was used in [6]. Using (20), we have the following representation of $A=\left(A_{1}, A_{2}\right)$ :

$$
A_{1}=-\frac{1}{4 \pi}\left(\frac{x_{2}}{|x|^{2}} *|\phi|^{2}\right), \quad A_{2}=\frac{1}{4 \pi}\left(\frac{x_{1}}{|x|^{2}} *|\phi|^{2}\right) .
$$

3.1. Estimates for $A$ and $A_{0}$. We are now ready to estimate several quantities of $A, A_{0}$. Making use of (20) and the representation (21), we obtain the following estimates for $A$.

Proposition 7. Let $s \geq 0$ and $0<2 / q<\delta<1$. One also assumes that $2 \leq p<\infty$ if $s>0$ or $2<p<\infty$ if $s=0$. Then, one has

$$
\begin{gathered}
\|\nabla A\|_{\dot{H}^{s}} \lesssim\|\phi\|_{L^{\infty}}\|\phi\|_{\dot{H}^{s}}, \\
\|\nabla A\|_{L^{\infty}} \lesssim\|\phi\|_{L^{\infty}}\left\|J^{\delta} \phi\right\|_{L^{q}}, \\
\|A\|_{L^{\infty}} \lesssim\|\phi\|_{L^{2}}\left\|J^{\delta} \phi\right\|_{L^{q}}, \\
\left\||\nabla|^{s} A\right\|_{L^{p}} \lesssim\|\phi\|_{L^{p}}\|\phi\|_{\dot{H}^{s}} .
\end{gathered}
$$

Proof. The above can be checked by applying CalderonZygmund and Hardy-Littlewood-Sobolev inequalities. We refer to [2, Section 2] for the details.

To estimate $A_{0}$, the special algebraic structure $Q_{12}$ and divergence form of the nonlinear terms in (19) are used.

Proposition 8. Let $A_{0}$ be the solution of (19). Then, one has

$$
\left\|A_{0}\right\|_{L^{\infty}}+\left\|\nabla A_{0}\right\|_{L^{2}} \lesssim\left(1+\|\phi\|_{L^{2}}^{2}\right)\|\nabla \phi\|_{L^{2}}^{2} .
$$

Proof. Decompose $A_{0}=A_{0}^{\prime}+A_{0}^{\prime \prime}$ as follows:

$$
\begin{gathered}
\Delta A_{0}^{\prime}=\operatorname{Im}\left(Q_{12}(\bar{\phi}, \phi)\right), \\
\Delta A_{0}^{\prime \prime}=\partial_{1}\left(A_{2}|\phi|^{2}\right)-\partial_{2}\left(A_{1}|\phi|^{2}\right) .
\end{gathered}
$$

We first estimate the quantity $\left\|A_{0}\right\|_{L^{\infty}\left(\mathbb{R}^{2}\right)}$. Applying Lemma 3 to (24), we deduce that

$$
\left\|A_{0}^{\prime}\right\|_{L^{\infty}} \lesssim\|\nabla \phi\|_{L^{2}}^{2}
$$

To estimate $\left\|A_{0}^{\prime \prime}\right\|_{L^{\infty}\left(\mathbb{R}^{2}\right)}$ we use the Gagliardo-Nirenberg inequality with small $\epsilon>0$ :

$$
\begin{array}{r}
\|u\|_{L^{\infty}\left(\mathbb{R}^{2}\right)} \leq C_{\epsilon}\|\Delta u\|_{L^{1+\epsilon}\left(\mathbb{R}^{2}\right)}^{\alpha}\|u\|_{L^{4}\left(\mathbb{R}^{2}\right)}^{1-\alpha}, \\
\text { with } \alpha=(1+\epsilon) /(1+5 \epsilon) .
\end{array}
$$

Applying Hardy-Littlewood-Sobolev's inequality to (25) we deduce

$$
\begin{aligned}
\left\|A_{0}^{\prime \prime}\right\|_{L^{4}} & \lesssim\left\|A|\phi|^{2}\right\|_{L^{4 / 3}} \lesssim\|A\|_{L^{4}}\|\phi\|_{L^{4}}^{2} \\
& \lesssim\|\phi\|_{L^{2}}\|\phi\|_{L^{4}}^{3} \lesssim\|\phi\|_{L^{2}}^{5 / 2}\|\nabla \phi\|_{L^{2}}^{3 / 2},
\end{aligned}
$$


where Proposition 7 and Lemma 6 are used. We can also derive the following from (25):

$$
\left\|\Delta A_{0}^{\prime \prime}\right\|_{L^{1+\epsilon}} \lesssim \underbrace{\left\|\nabla A \cdot|\phi|^{2}\right\|_{L^{1+\epsilon}}}_{(\mathrm{i})}+\underbrace{\left\|A \nabla\left(|\phi|^{2}\right)\right\|_{L^{1+\epsilon}}}_{(\mathrm{ii})} .
$$

The first term can be estimated as follows:

$$
(\mathrm{i}) \lesssim\|\nabla A\|_{L^{2}}\left\|\phi^{2}\right\|_{L^{(2+2 \epsilon) /(1-\epsilon)}} \lesssim\|\phi\|_{L^{2}}^{2 /(1+\epsilon)}\|\nabla \phi\|_{L^{2}}^{(2+4 \epsilon) /(1+\epsilon)},
$$

where $\|\phi\|_{L^{(4+4 \epsilon) /(1-\epsilon)}} \lesssim\|\phi\|_{L^{2}}^{(1-\epsilon) /(2+2 \epsilon)}\|\nabla \phi\|_{L^{2}}^{(1+3 \epsilon) /(2+2 \epsilon)}$ is used. The second term can be estimated as follows:

$$
\begin{aligned}
\text { (ii) } & \lesssim\|A\|_{L^{4}}\|\phi\|_{L^{(4+4 \epsilon) /(1-3 \epsilon)}\|\nabla \phi\|_{L^{2}}} \\
& \lesssim\|\phi\|_{L^{2}}\|\phi\|_{L^{4}}\|\phi\|_{L^{2}}^{(1-3 \epsilon) /(2+2 \epsilon)}\|\nabla \phi\|_{L^{2}}^{(3+7 \epsilon) /(2+2 \epsilon)} \\
& \lesssim\|\phi\|_{L^{2}}^{2 /(1+\epsilon)}\|\nabla \phi\|_{L^{2}}^{(2+4 \epsilon) /(1+\epsilon)},
\end{aligned}
$$

where $\|\phi\|_{L^{(4+4 \epsilon) /(1-3 \epsilon)}} \lesssim\|\phi\|_{L^{2}}^{(1-3 \epsilon) /(2+2 \epsilon)}\|\nabla \phi\|_{L^{2}}^{(1+5 \epsilon) /(2+2 \epsilon)}$ is used. Therefore, we obtain with $\epsilon=1 / 11$, that is, $\alpha=3 / 4$,

$$
\left\|A_{0}^{\prime \prime}\right\|_{L^{\infty}} \lesssim\|\phi\|_{L^{2}}^{2}\|\nabla \phi\|_{L^{2}}^{2}
$$

Therefore, we conclude that

$$
\left\|A_{0}\right\|_{L^{\infty}} \lesssim\|\nabla \phi\|_{L^{2}}^{2}\left(1+\|\phi\|_{L^{2}}^{2}\right) .
$$

On the other hand, Lemma 3 shows that

$$
\left\|\nabla A_{0}^{\prime}\right\|_{L^{2}} \lesssim\|\nabla \phi\|_{L^{2}}^{2}
$$

We also have from (25) that

$$
\begin{aligned}
\left\|\nabla A_{0}^{\prime \prime}\right\|_{L^{2}} & \lesssim\left\|A|\phi|^{2}\right\|_{L^{2}} \lesssim\|A\|_{L^{4}}\|\phi\|_{L^{8}}^{2} \\
& \lesssim\|\phi\|_{L^{2}}\|\phi\|_{L^{4}}\|\phi\|_{L^{8}}^{2} \lesssim\|\phi\|_{L^{2}}^{2}\|\nabla \phi\|_{L^{2}}^{2} .
\end{aligned}
$$

Therefore, we have

$$
\left\|\nabla A_{0}\right\|_{L^{2}} \lesssim\|\nabla \phi\|_{L^{2}}^{2}\left(1+\|\phi\|_{L^{2}}^{2}\right) .
$$

3.2. The Energy Solution to (CSS). We now prove Theorem 1. Let us define

$$
X(T)=\|\phi\|_{L_{T}^{\infty} H^{1}}+\left\|J^{\delta} \phi\right\|_{L_{T}^{p} L^{q}},
$$

where $0<\delta<1 / 2,2<\delta q$, and $1 / p+1 / q=1 / 2$. We derive the following estimate:

$$
X \lesssim\left\|\phi_{0}\right\|_{H^{1}}+T^{1 / 6}\left\|\phi_{0}\right\|_{L^{2}}\left(1+\left\|\phi_{0}\right\|_{L^{2}}^{2}\right)\left(X^{2}+X^{4}\right),
$$

from which Theorem 1 is proved by standard argument; see [2, Section 3].

To control $\|\phi\|_{L_{T}^{\infty} H^{1}}$, we apply Lemma 4 to the solution of (6)-(8).
Proposition 9. Let $\phi$ be a solution to (6)-(8). Then, one has $\|\phi\|_{L_{T}^{\infty} L^{2}}=\left\|\phi_{0}\right\|_{L^{2}}$

$\|\phi\|_{L_{T}^{\infty} \dot{H}^{1}} \lesssim\left\|\phi_{0}\right\|_{\dot{H}^{1}}$

$$
+\left(1+\left\|\phi_{0}\right\|_{L^{2}}^{2}\right) T^{(p-3) / p}\left(\|\phi\|_{L_{T}^{\infty} \dot{H}^{1}}^{3}+\left\|J^{\delta} \phi\right\|_{L_{T}^{p} L^{q}}^{3}\right),
$$

where $2<\delta q$ and $3<p<\infty$.

Proof. From the conservation of mass, we derive the first estimate. We apply Lemma 4 to (6) with $F=A_{0} \phi+A_{j}^{2} \phi-$ $\lambda|\phi|^{2} \phi$ and $s=1$. Combined with Proposition 7, we have

$$
\begin{gathered}
\|\nabla A\|_{\dot{H}^{1}}\|\phi\|_{L^{\infty}} \lesssim\|\phi\|_{W^{\delta, q}}^{2}\|\phi\|_{\dot{H}^{1}}, \\
\|\nabla A\|_{L^{\infty}}\|\phi\|_{\dot{H}^{1}} \lesssim\|\phi\|_{W^{\delta, q}}^{2}\|\phi\|_{\dot{H}^{1}}, \\
\left\|A^{2} \phi\right\|_{\dot{H}^{1}} \lesssim\|\phi\|_{L^{2}}^{2}\left(\|\phi\|_{W^{\delta, q}}^{3}+\|\phi\|_{\dot{H}^{1}}^{3}\right), \\
\left\||\phi|^{2} \phi\right\|_{\dot{H}^{1}} \lesssim\|\phi\|_{W^{\delta, q}}^{2}\|\phi\|_{\dot{H}^{1}},
\end{gathered}
$$

where $2<\delta q$. We are then left to estimate $\left\|A_{0} \phi\right\|_{\dot{H}^{1}}$. By Proposition 8, we obtain

$$
\begin{aligned}
\left\|A_{0} \phi\right\|_{\dot{H}^{1}} & \leq\left\|A_{0}\right\|_{L^{\infty}}\|\phi\|_{\dot{H}^{1}}+\left\|A_{0}\right\|_{\dot{H}^{1}}\|\phi\|_{L^{\infty}} \\
& \leq\left(1+\|\phi\|_{L^{2}}^{2}\right)\left(\|\nabla \phi\|_{L^{2}}^{3}+\left\|J^{\delta} \phi\right\|_{L^{q}}^{3}\right) .
\end{aligned}
$$

Combining (40) and (41), we obtain

$$
\begin{aligned}
\|\phi\|_{L_{T}^{\infty} \dot{H}^{1}} \lesssim & \left\|\phi_{0}\right\|_{\dot{H}^{1}}+\int_{0}^{T}\left(1+\left\|\phi_{0}\right\|_{L^{2}}^{2}\right)\left(\|\phi\|_{\dot{H}^{1}}^{3}+\left\|J^{\delta} \phi\right\|_{L^{q}}^{3}\right) \\
\lesssim & \left\|\phi_{0}\right\|_{\dot{H}^{1}}+\left(1+\left\|\phi_{0}\right\|_{L^{2}}^{2}\right) T^{(p-3) / p} \\
& \times\left(\|\phi\|_{L_{T}^{\infty} \dot{H}^{1}}^{3}+\left\|J^{\delta} \phi\right\|_{L_{T}^{p} L^{q}}^{3}\right),
\end{aligned}
$$

where $3<p<\infty$ and $T<1$.

To estimate $\left\|J^{\delta} \phi\right\|_{L_{T}^{p} L^{q}}$, we apply Lemma 5 to the solution of (6)-(8).

Proposition 10. Let $\phi$ be a solution to (6)-(8). Then, one has

$$
\left\|J^{\delta} \phi\right\|_{L_{T}^{p} L^{q}} \lesssim\|\phi\|_{L_{T}^{\infty} H^{1}}+T^{1 / 6}\left\|\phi_{0}\right\|_{L^{2}}\left(1+\left\|\phi_{0}\right\|_{L^{2}}^{2}\right)\left(X^{2}+X^{4}\right),
$$

where $2<\delta q, 3<p<\infty$ and $1 / p+1 / q=1 / 2$.

Proof. Applying Lemma 5 with $F_{1}=A_{0} \phi-2 i A_{j} \partial_{j} \phi$ and $F_{2}=$ $A^{2} \phi-\lambda|\phi|^{2} \phi$, we obtain

$$
\begin{aligned}
\left\|J^{\delta} \phi\right\|_{L_{T}^{p} L^{q}} \lesssim & \|\phi\|_{L_{T}^{\infty} H^{1}}+\left\|A_{0} \phi\right\|_{L_{T}^{2} H^{\delta-1 / 2}} \\
& +\|A \cdot \nabla \phi\|_{L_{T}^{2} H^{\delta-1 / 2}}+\left\|A^{2} \phi\right\|_{L_{T}^{1} H^{\delta}}+\left\||\phi|^{2} \phi\right\|_{L_{T}^{1} H^{\delta}},
\end{aligned}
$$


where $\delta=1 / 2-\varepsilon, 3<p<\infty$ and $2<\delta q$. Considering Proposition 8 , we obtain

$$
\begin{aligned}
& \left\|A_{0} \phi\right\|_{L_{T}^{2} H^{\delta-1 / 2}} \\
& \quad \leq\left\|A_{0}\right\|_{L_{T}^{\infty} L^{\infty}}\|\phi\|_{L_{T}^{2} L^{2}} \leqslant T^{1 / 2}\left\|\phi_{0}\right\|_{L^{2}}\left(1+\left\|\phi_{0}\right\|_{L^{2}}^{2}\right)\|\nabla \phi\|_{L^{2}}^{2} .
\end{aligned}
$$

The other terms can be treated, as mentioned in Section 1, by similar arguments to those in [2, Section 3]. Applying Proposition 7, we have

$$
\begin{aligned}
\|A \cdot \nabla \phi\|_{L_{T}^{2} H^{\delta-1 / 2}} \leq & \|A\|_{L_{T}^{2} L^{\infty}}\|\nabla \phi\|_{L_{T}^{\infty} L^{2}} \\
\leq & \left\|\phi_{0}\right\|_{L^{2}} T^{(p-2) / 2 p}\left\|J^{\delta} \phi\right\|_{L_{T}^{p} L^{q}}\|\phi\|_{L_{T}^{\infty} H^{1}}, \\
\left\|A^{2} \phi\right\|_{L_{T}^{1} H^{\delta}} \leq & \left\|A^{2}\right\|_{L_{T}^{1} L^{L}}\left\|J^{\delta} \phi\right\|_{L_{T}^{\infty} L^{4}} \\
& +\left\|A^{2}\right\|_{L_{T}^{2} W^{\delta, 2+\varepsilon}}\|\phi\|_{L_{T}^{2} L^{(4+2 \varepsilon) / \varepsilon}} \\
\leq & T\left\|\phi_{0}\right\|_{L^{2}}^{3 / 2}\|\phi\|_{L_{T}^{\infty} H^{1}}^{5 / 2} \\
& +T^{1 / 4}\left\|\phi_{0}\right\|_{L^{2}}\|\phi\|_{L_{T}^{\infty} H^{1}}^{2}\left\|J^{\delta} \phi\right\|_{L_{T}^{p} L^{q}}^{2} \\
\left\|\phi^{3}\right\|_{L_{T}^{1} H^{\delta}} \leq & \left\|J^{\delta} \phi\right\|_{L_{T}^{\infty} L^{L^{\prime}}}\|\phi\|_{L_{T}^{2} L^{\infty}}^{2} \\
\leq & T^{(p-2) / p}\|\phi\|_{L_{T}^{\infty} H^{1}}\left\|J^{\delta} \phi\right\|_{L_{T}^{p} L^{q}}^{2}
\end{aligned}
$$

Plugging estimates (45)-(48) into (44) with $p>3$, we obtain

$$
\left\|J^{\delta} \phi\right\|_{L_{T}^{p} L^{q}} \lesssim\|\phi\|_{L_{T}^{\infty} H^{1}}+T^{1 / 6}\left\|\phi_{0}\right\|_{L^{2}}\left(1+\left\|\phi_{0}\right\|_{L^{2}}^{2}\right)\left(X^{2}+X^{4}\right) .
$$

We finally obtain the estimate (38) by combining Propositions 9 and 10, which proves Theorem 1 .

\section{The Proof of Theorem 2}

In this section, we prove the uniqueness of the solution to (6). The basic rationale is borrowed from $[12,22]$.

Let $\left(\phi, A_{0}, A\right)$ and $\left(\psi, B_{0}, B\right)$ be solutions of (6)-(8) with the same initial data. If we set $\omega=\phi-\psi$, then the equation for $\omega$ is

$$
\begin{aligned}
i \partial_{t} \omega+\Delta \omega= & A_{0} \omega+\left(A_{0}-B_{0}\right) \psi-2 i A \cdot \nabla \omega-2 i(A-B) \cdot \nabla \psi \\
& +A^{2} \omega+\left(A^{2}-B^{2}\right) \psi-\lambda|\phi|^{2} \omega-\lambda\left(|\phi|^{2}-|\psi|^{2}\right) \psi .
\end{aligned}
$$

We will derive

$$
\partial_{t}\|\omega\|_{L^{2}}^{2} \lesssim q^{1 / 2} M^{2}\|\omega\|_{L^{2}}^{2-4 / q}+q M^{2+4 / q}\left(1+M^{2}\right)\|\omega\|_{L^{2}}^{2-4 / q},
$$

where $M$ is a constant in Theorem 2 and $q>2$. Then we have

$$
\partial_{t}\|\omega\|_{L^{2}}^{4 / q} \lesssim \frac{1}{q}\left(q^{1 / 2} M^{2}+q M^{2+4 / q}\left(1+M^{2}\right)\right) .
$$

Considering $\|\omega(0, \cdot)\|_{L^{2}}=0$ and $2<q$, we obtain

$$
\|\omega\|_{L^{2}} \lesssim\left(T\left(M^{2}+M^{4+4 / q}\right)\right)^{q / 4} .
$$

Letting $q \rightarrow \infty$, for the time interval satisfying $T\left(M^{2}+\right.$ $\left.M^{4+4 / q}\right) \leq 1 / 2$, we conclude that $\|\omega(t, \cdot)\|_{L^{2}}=0$ for $0 \leq t \leq T$, which thus proves Theorem 2 .

In the remainder of this section, we derive inequality (51). Multiplying $\bar{\omega}$ to both sides of (50) and integrating the imaginary part of $\mathbb{R}^{2}$, we have

$$
\begin{aligned}
\partial_{t}\|\omega\|_{L^{2}}^{2}=\int & \underbrace{2\left(A_{0}-B_{0}\right) \operatorname{Im}(\psi \bar{\omega})}_{(\mathrm{I})}-\underbrace{2 A_{j} \partial_{j}|\omega|^{2}}_{(\mathrm{II})} \\
& -\underbrace{4\left(A_{j}-B_{j}\right) \operatorname{Re}\left(\partial_{j} \psi \bar{\omega}\right)}_{(\mathrm{III})} d x \\
& +\int \underbrace{2\left(A^{2}-B^{2}\right) \operatorname{Im}(\psi \bar{\omega})}_{(\mathrm{IV})} \\
& -\underbrace{2 \lambda\left(|\phi|^{2}-|\psi|^{2}\right) \operatorname{Im}(\psi \bar{\omega})}_{(\mathrm{V})} d x .
\end{aligned}
$$

The integrals (II)-(V), that is, those not containing $A_{0}$, can be controlled by applying similar arguments to those described in [2, Section 4]. Integral (II) can be estimated, considering $\partial_{j} A_{j}=0$, by

$$
\begin{gathered}
\int-A_{j} \partial_{j}|\omega|^{2} d x=\int \partial_{j} A_{j}|\omega|^{2} d x=0, \\
(\mathrm{III}),(\mathrm{IV}),(\mathrm{V}) \leqslant q M^{2+4 / q}\left(1+M^{2}\right)\|\omega\|_{L^{2}}^{2-4 / q}
\end{gathered}
$$

for which we omit the proof.

We simply present how to control integral (I), for which we have

$$
\left|\int\left(A_{0}-B_{0}\right) \operatorname{Im}(\psi \bar{\omega}) d x\right| \leqslant\left\|A_{0}-B_{0}\right\|_{L^{a}}\|\psi\|_{L^{b}}\|\omega\|_{L^{c}},
$$

where $1 / a+1 / b+1 / c=1,2 \leq a, b, c$. Applying Lemma 6 , we obtain

$$
\begin{gathered}
\|\psi\|_{L^{b}} \lesssim b^{1 / 2}\|\psi\|_{L^{2}}^{2 / b}\|\nabla \psi\|_{L^{2}}^{1-2 / b} \lesssim b^{1 / 2} M^{1-2 / b} \\
\|\omega\|_{L^{c}} \lesssim c^{1 / 2}\|\omega\|_{L^{2}}^{2 / c}\|\nabla \omega\|_{L^{2}}^{1-2 / c} \lesssim c^{1 / 2}\|\omega\|_{L^{2}}^{2 / c} M^{1-2 / c} .
\end{gathered}
$$

To control $\left\|A_{0}-B_{0}\right\|_{L^{a}}$, we consider the equation for $A_{0}-B_{0}$

$$
\begin{aligned}
\Delta\left(A_{0}-B_{0}\right)= & \partial_{1} \operatorname{Im}\left(\bar{\phi} \partial_{2} \phi\right)-\partial_{2} \operatorname{Im}\left(\bar{\phi} \partial_{1} \phi\right)-\partial_{1} \operatorname{Im}\left(\bar{\psi} \partial_{2} \psi\right) \\
& +\partial_{2} \operatorname{Im}\left(\bar{\psi} \partial_{1} \psi\right)+\partial_{1}\left(A_{2}|\phi|^{2}\right)-\partial_{2}\left(A_{1}|\phi|^{2}\right) \\
& -\partial_{1}\left(B_{2}|\psi|^{2}\right)+\partial_{2}\left(B_{1}|\psi|^{2}\right) .
\end{aligned}
$$


Decomposing $A_{0}$ and $B_{0}$ as (24) and (25), we have

$$
\begin{aligned}
\Delta\left(A_{0}^{\prime}-B_{0}^{\prime}\right)= & \partial_{1} \operatorname{Im}\left(\bar{\phi} \partial_{2} \omega\right)-\partial_{2} \operatorname{Im}\left(\bar{\psi} \partial_{1} \omega\right) \\
& +\partial_{1} \operatorname{Im}\left(\bar{\omega} \partial_{2} \psi\right)-\partial_{2} \operatorname{Im}\left(\bar{\omega} \partial_{1} \phi\right), \\
\Delta\left(A_{0}^{\prime \prime}-B_{0}^{\prime \prime}\right)= & \partial_{1}\left(A_{2}\left(|\phi|^{2}-|\psi|^{2}\right)\right)-\partial_{2}\left(A_{1}\left(|\phi|^{2}-|\psi|^{2}\right)\right) \\
& +\partial_{1}\left(\left(A_{2}-B_{2}\right)|\psi|^{2}\right)-\partial_{2}\left(\left(A_{1}-B_{1}\right)|\phi|^{2}\right) .
\end{aligned}
$$

Taking into account

$$
\begin{aligned}
& \partial_{1} \operatorname{Im}\left(\bar{\phi} \partial_{2} \omega\right)=\partial_{1}\left(\partial_{2} \operatorname{Im}(\bar{\phi} \omega)-\operatorname{Im}\left(\omega \partial_{2} \bar{\phi}\right)\right), \\
& \partial_{2} \operatorname{Im}\left(\bar{\psi} \partial_{1} \omega\right)=\partial_{2}\left(\partial_{1} \operatorname{Im}(\bar{\psi} \omega)-\operatorname{Im}\left(\omega \partial_{1} \bar{\psi}\right)\right),
\end{aligned}
$$

we can rewrite the equation for $A_{0}^{\prime}-B_{0}^{\prime}$ as follows:

$$
\begin{aligned}
\Delta\left(A_{0}^{\prime}-B_{0}^{\prime}\right)= & \partial_{1}\left(\operatorname{Im}\left(\bar{\omega} \partial_{2} \psi\right)-\operatorname{Im}\left(\omega \partial_{2} \bar{\phi}\right)\right) \\
& +\partial_{2}\left(\operatorname{Im}\left(\omega \partial_{1} \bar{\psi}\right)-\operatorname{Im}\left(\bar{\omega} \partial_{2} \phi\right)\right),
\end{aligned}
$$

where $\partial_{1} \partial_{2} \operatorname{Im}(\bar{\phi} \omega)-\partial_{2} \partial_{1} \operatorname{Im}(\bar{\psi} \omega)=\partial_{1} \partial_{2} \operatorname{Im}(\bar{\omega} \omega)=0$ should be noted. Using the Hardy-Littlewood-Sobolev inequality, we have

$$
\begin{aligned}
\left\|A_{0}^{\prime}-B_{0}^{\prime}\right\|_{L^{a}} & \lesssim\left\||x|^{-1} *(\omega \nabla \psi)\right\|_{L^{a}} \\
& \lesssim\|\omega \nabla \psi\|_{L^{r}} \lesssim\|\omega\|_{L^{s}}\|\nabla \psi\|_{L^{2}},
\end{aligned}
$$

where $1 / a=1 / r-1 / 2$ and $1 / r=1 / s+1 / 2$, from which we deduce $a=s$. Then, we have

$$
\|\omega\|_{L^{a}}\|\nabla \psi\|_{L^{2}} \lesssim a^{1 / 2}\|\omega\|_{L^{2}}^{2 / a}\|\nabla \omega\|_{L^{2}}^{1-2 / a} M \lesssim a^{1 / 2} M^{2-2 / a}\|\omega\|_{L^{2}}^{2 / a} .
$$

The term $A_{0}^{\prime \prime}-B_{0}^{\prime \prime}$ can be bounded as follows:

$$
\begin{aligned}
\left\|A_{0}^{\prime \prime}-B_{0}^{\prime \prime}\right\|_{L^{a}} \leqslant & \underbrace{\left\||x|^{-1} *\left(\left.|A||| \phi\right|^{2}-|\psi|^{2} \mid\right)\right\|_{L^{a}}}_{(1)} \\
& +\underbrace{\left\||x|^{-1} *\left(|A-B|\left(|\phi|^{2}+|\psi|^{2}\right)\right)\right\|_{L^{a}}}_{(2)} .
\end{aligned}
$$

Since $\left.|| \phi\right|^{2}-|\psi|^{2}|\leq(|\phi|+|\psi|)| \omega \mid$, we have

$$
\begin{aligned}
(1) & \lesssim\||A|(|\phi|+|\psi|)\|_{L^{2}}\|\omega\|_{L^{a}} \\
& \lesssim\|A\|_{L^{6}}\left(\|\phi\|_{L^{3}}+\|\psi\|_{L^{3}}\right)\|\omega\|_{L^{a}} \\
& \lesssim\|\phi\|_{L^{3}}^{2}\left(\|\phi\|_{L^{3}}+\|\psi\|_{L^{3}}\right)\|\omega\|_{L^{a}} \\
& \lesssim a^{1 / 2} M^{2-2 / a}\|\omega\|_{L^{2}}^{2 / a} .
\end{aligned}
$$

Since $\left|A_{j}-B_{j}\right| \lesssim|x|^{-1} *((|\phi|+|\psi|)|\omega|)$, we may check

$$
\begin{aligned}
(2) & \lesssim\left\|A_{j}-B_{j}\right\|_{L^{a}}\left(\left\|\phi^{2}\right\|_{L^{2}}+\left\|\psi^{2}\right\|_{L^{2}}\right) \\
& \leqslant\left(\|\phi\|_{L^{2}}+\|\psi\|_{L^{2}}\right)\|\omega\|_{L^{a}}\left(\|\phi\|_{L^{4}}^{2}+\|\psi\|_{L^{4}}^{2}\right) \\
& \lesssim a^{1 / 2}\|\omega\|_{L^{2}}^{2 / a}\|\nabla \omega\|_{L^{2}}^{1-2 / a}\left(\|\nabla \phi\|_{L^{2}}+\|\nabla \psi\|_{L^{2}}\right) \\
& \leqslant a^{1 / 2} M^{2-2 / a}\|\omega\|_{L^{2}}^{2 / a} .
\end{aligned}
$$

Then, we have

$$
\left\|A_{0}-B_{0}\right\|_{L^{a}} \lesssim a^{1 / 2} M^{2-2 / a}\|\omega\|_{L^{2}}^{2 / a} .
$$

Combining estimates (57) and (69), and denoting $b=q / 2$, we obtain

$$
\left\|A_{0}-B_{0}\right\|_{L^{a}}\|\psi\|_{L^{b}}\|\omega\|_{L^{c}} \lesssim(a q c)^{1 / 2} M^{2}\|\omega\|_{L^{2}}^{2-4 / q},
$$

where $1 / a+2 / q+1 / c=1$. We then obtain (51) by combining (55) and (70).

\section{Acknowledgments}

This research was supported by the Basic Science Research Program through the National Research Foundation of Korea (NRF), funded by the Ministry of Education, Science and Technology (2011-0015866), and was also partially supported by the TJ Park Junior Faculty Fellowship.

\section{References}

[1] R. Jackiw and S.-Y. Pi, "Classical and quantal nonrelativistic Chern-Simons theory," Physical Review D, vol. 42, no. 10, pp. 3500-3513, 1990.

[2] R. Jackiw and S.-Y. Pi, "Self-dual Chern-Simons solitons," Progress of Theoretical Physics. Supplement, no. 107, pp. 1-40, 1992.

[3] G. Dunne, Self-Dual Chern-Simons Theories, Springer, Berlin, Germany, 1995.

[4] P. A. Horvathy and P. Zhang, "Vortices in (abelian) ChernSimons gauge theory," Physics Reports, vol. 481, no. 5-6, pp. 83$142,2009$.

[5] K. Nakamitsu and M. Tsutsumi, "The Cauchy problem for the coupled Maxwell-Schrödinger equations," Journal of Mathematical Physics, vol. 27, no. 1, pp. 211-216, 1986.

[6] L. Bergé, A. de Bouard, and J.-C. Saut, "Blowing up timedependent solutions of the planar, Chern-Simons gauged nonlinear Schrödinger equation," Nonlinearity, vol. 8, no. 2, pp. 235253, 1995.

[7] S. Demoulini, "Global existence for a nonlinear SchroedingerChern-Simons system on a surface," Annales de l'Institut Henri Poincaré. Analyse Non Linéaire, vol. 24, no. 2, pp. 207-225, 2007.

[8] H. Huh, "Blow-up solutions of the Chern-Simons-Schrödinger equations," Nonlinearity, vol. 22, no. 5, pp. 967-974, 2009.

[9] J. Byeon, H. Huh, and J. Seok, "Standing waves of nonlinear Schrödinger equations with the gauge field," Journal of Functional Analysis, vol. 263, no. 6, pp. 1575-1608, 2012.

[10] H. Huh, "Standing waves of the Schrödinger equation coupled with the Chern-Simons gauge field," Journal of Mathematical Physics, vol. 53, no. 6, p. 063702, 8, 2012.

[11] S. Demoulini and D. Stuart, "Adiabatic limit and the slow motion of vortices in a Chern-Simons-Schrödinger system," Communications in Mathematical Physics, vol. 290, no. 2, pp. 597-632, 2009.

[12] J. Kato, "Existence and uniqueness of the solution to the modified Schrödinger map," Mathematical Research Letters, vol. 12, no. 2-3, pp. 171-186, 2005.

[13] C. E. Kenig and A. R. Nahmod, "The Cauchy problem for the hyperbolic-elliptic Ishimori system and Schrödinger maps," Nonlinearity, vol. 18, no. 5, pp. 1987-2009, 2005. 
[14] J. Kato and H. Koch, "Uniqueness of the modified Schrödinger map in $H^{3 / 4+\epsilon}\left(\mathbb{R}^{2}\right)$," Communications in Partial Differential Equations, vol. 32, no. 1-3, pp. 415-429, 2007.

[15] H. Brezis and J.-M. Coron, "Multiple solutions of $H$-systems and Rellich's conjecture," Communications on Pure and Applied Mathematics, vol. 37, no. 2, pp. 149-187, 1984.

[16] H. C. Wente, "An existence theorem for surfaces of constant mean curvature," Journal of Mathematical Analysis and Applications, vol. 26, pp. 318-344, 1969.

[17] A. Nahmod, A. Stefanov, and K. Uhlenbeck, "On Schrödinger maps," Communications on Pure and Applied Mathematics, vol. 56, no. 1, pp. 114-151, 2003.

[18] A. Nahmod, A. Stefanov, and K. Uhlenbeck, "Erratum: on Schrödinger maps," Communications on Pure and Applied Mathematics, vol. 57, no. 6, pp. 833-839, 2004.

[19] C. E. Kenig and K. D. Koenig, "On the local well-posedness of the Benjamin-Ono and modified Benjamin-Ono equations," Mathematical Research Letters, vol. 10, no. 5-6, pp. 879-895, 2003.

[20] H. Koch and N. Tzvetkov, "On the local well-posedness of the Benjamin-Ono equation in $H^{s}(\mathbb{R})$," International Mathematics Research Notices, no. 26, pp. 1449-1464, 2003.

[21] T. Ogawa, "A proof of Trudinger's inequality and its application to nonlinear Schrödinger equations," Nonlinear Analysis. Theory, Methods \& Applications, vol. 14, no. 9, pp. 765-769, 1990.

[22] M. V. Vladimirov, "On the solvability of a mixed problem for a nonlinear equation of Schrödinger type," Doklady Akademii Nauk SSSR, vol. 275, no. 4, pp. 780-783, 1984. 


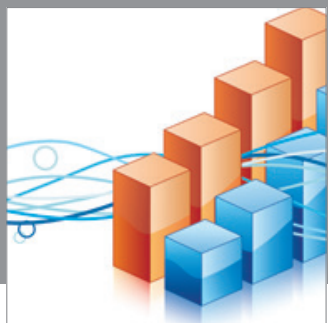

Advances in

Operations Research

mansans

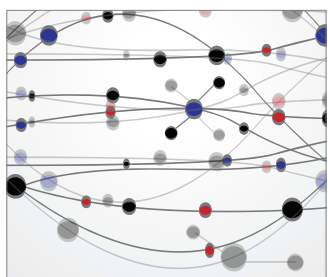

The Scientific World Journal
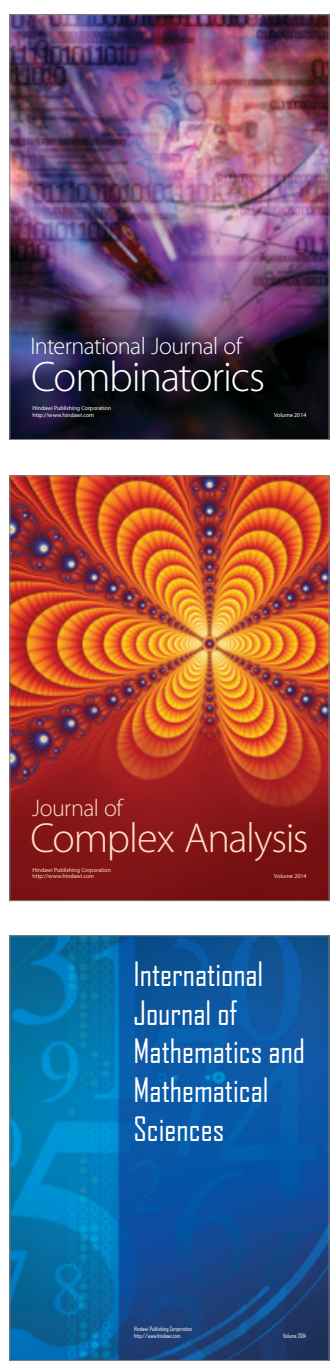
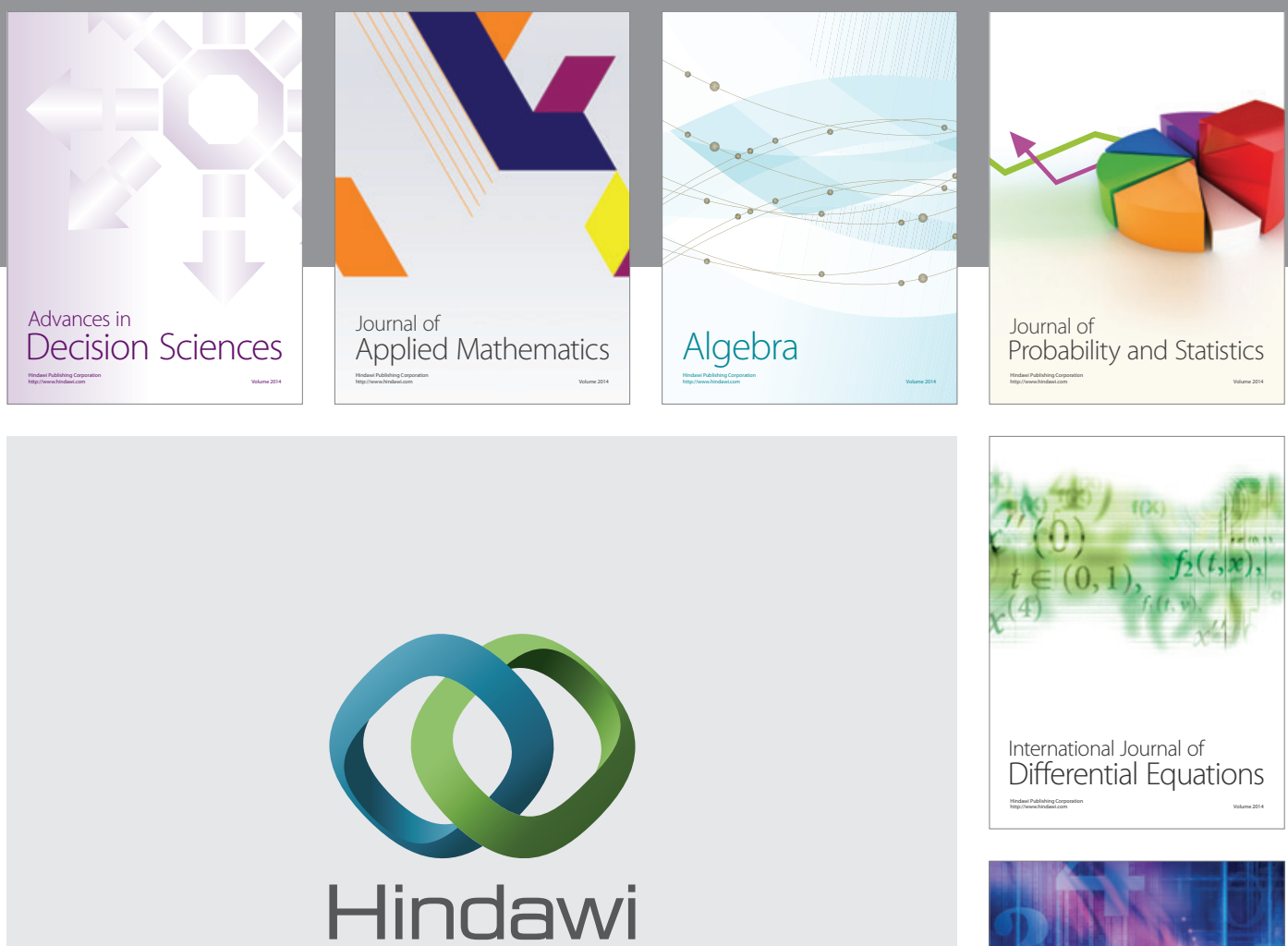

Submit your manuscripts at http://www.hindawi.com
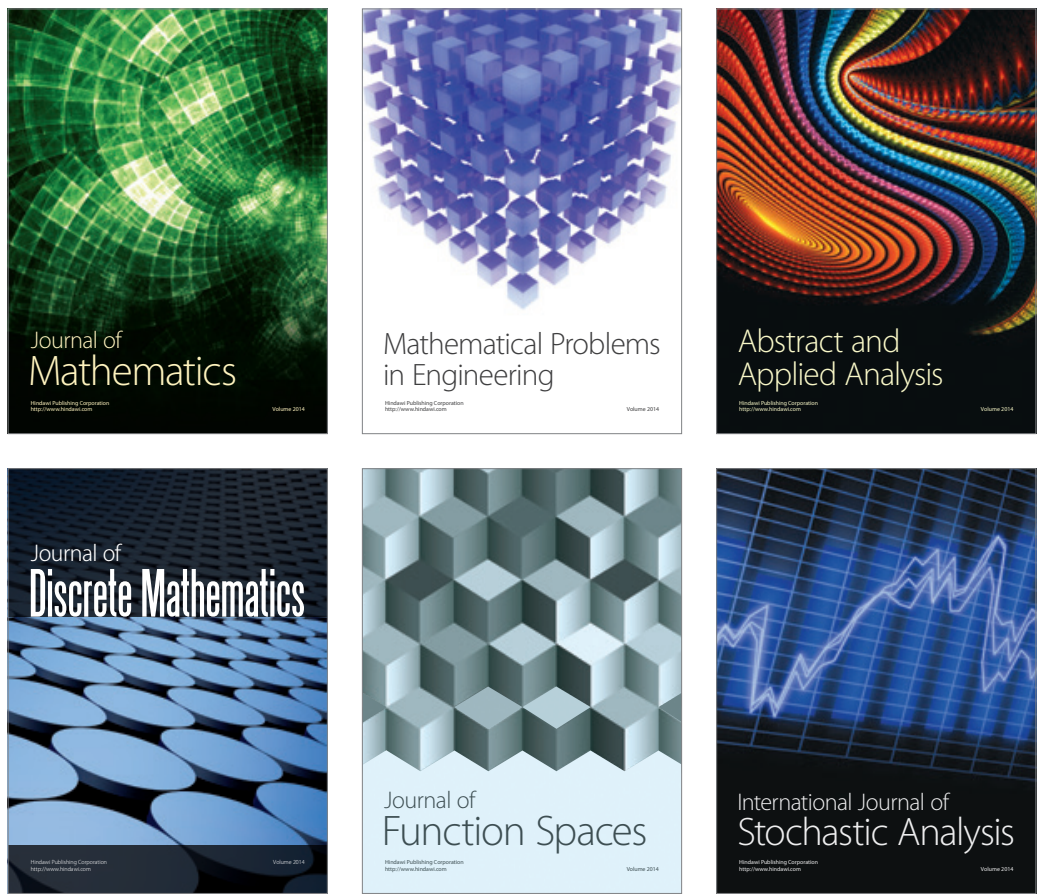

Journal of

Function Spaces

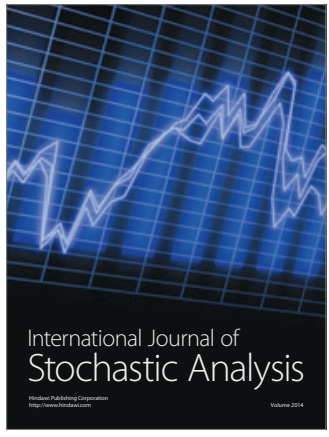

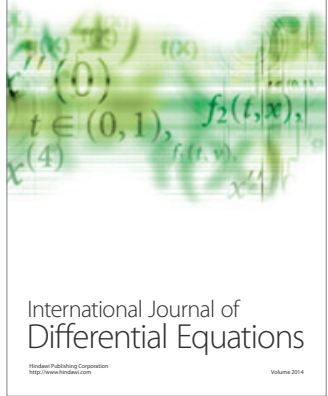
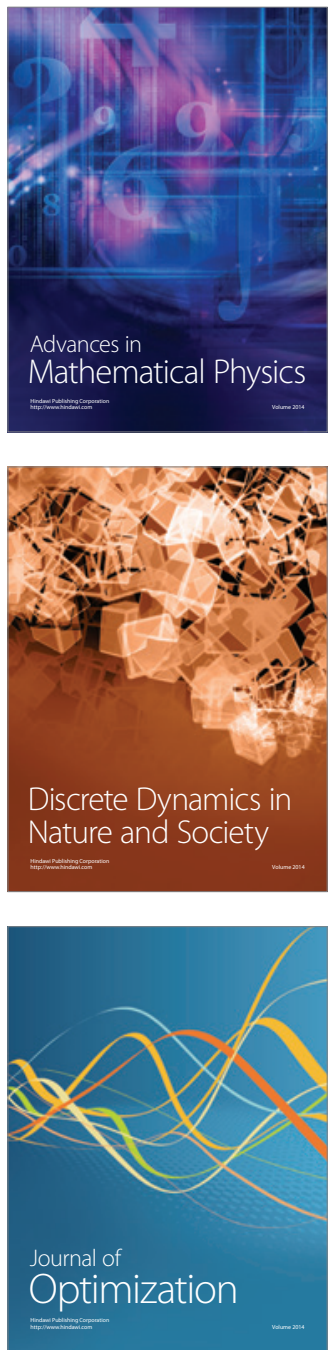